\title{
LARGE-SCALE INTERNAL MAGNETIC FIELD OF THE SUN
}

\author{
A.E. DUDOROV ${ }^{1}$, V.N. KRIVODUBSKIJ ${ }^{2}$, A.A. RUZMAIKIN ${ }^{3}$, \\ and T.V. RUZMAIKINA ${ }^{4}$ \\ ${ }^{1}$ Astronomy Department of Moscow State University \\ 117899, Moscow, USSR \\ ${ }^{2}$ Kiev Shevchenko University Astronomical Observatory \\ 252053, Kiev, USSR \\ ${ }^{3}$ IZMIRAN, 142092, Troitsk, Moscow region, USSR \\ ${ }^{4}$ Institute of Physics of Earth, 123810, Moscow, USSR
}

\begin{abstract}
ABS'RRACT. The behaviour of the magnetic field during the formation and evolution of the Sun is investigated. It is shown that an internal poloidal magnetic field of the order of $10^{4}-10^{5} \mathrm{G}$ near the core of the Sun may be compatible with differential rotation and with torsional waves, travelling along the magnetic field lines (Dudorov et al., 1989).
\end{abstract}

\section{The fossil magnetic field}

Estimates and numerical calculations reveal an intensification of the magnetic field during the contraction of the magnetized protosolar cloud, in spite of some loss of magnetic flux (Ruzmaikina, 1981, 1985; Dudorov, 1986; Dudorov and Sazonov, 1981).

The fossil magnetic field strength of the young Sun has been calculated using a computer code for protostar evolution (Dudorov and Sazonov, 1981). The initial model is a uniform cloud of mass $M=1.2 M_{\odot}$, temperature $T_{0}=10 \mathrm{~K}$, density $n_{0}=2 \times 10^{6} \mathrm{~cm}^{-3}$. The cloud is permeated by a homogeneous magnetic field of strength $B=4.5 \times 10^{-5} \mathrm{G}$. The evolution of the magnetic flux is investigated in the framework of the kinematic problem for collapse of protostellar clouds. The computer code based on the Lax-Wendroff method solves the gas-dynamic equations together with the induction equation:

$$
\mathrm{d} \mathbf{B} / \mathrm{d} t=\nabla \times\left[\left(\mathbf{V}-\mathbf{V}_{A D}\right) \times \mathbf{B}\right]+(\mathbf{V} \cdot \nabla) \mathbf{B}-\nabla \times\left(\nu_{m} \nabla \times \mathbf{B}\right),
$$

where $\mathbf{V}=\left\{V_{r}, 0,0\right\}$ is the gas velocity, $\mathbf{B}=\left\{B_{r} \cos \theta,-B_{\theta} \sin \theta, 0\right\}$ the strength of the magnetic field, $\nu_{m}$ the magnetic viscosity, and $\mathbf{V}_{A D}=3.6 \times 10^{31}<\operatorname{curl} \mathrm{B} \times \mathrm{B}>/ n^{2} / x$ $\left(\mathrm{cm} \mathrm{s}^{-1}\right)$ the velocity of ambipolar diffusion. The calculations of ionization degree $x$ take into account the collisional and thermal ionization of elements with sufficiently high cosmic abundances and low ionization potentials. The total ionization rate is determined by the collisional and radiative rates, and by radioactive elements. The recombination processes are radiative recombination and recombination on grains. 
The calculations show that during the collapse the field strength changes as $B \sim n^{k}$ with $1 / 2<k<2 / 3$. When an opaque core in the cloud is formed with $n_{i}>10^{5} n_{0}$, the ionization ratio is decreased to $x<10^{-12}-10^{-13}$. The coupling between plasma and neutrals weakens, giving rise to ambipolar diffusion of the charged particles with the magnetic field in the neutral gas. An upper limit to the effective flux loss in the dense $\left(n=10^{9} n_{0}\right)$ region is determined by the evaporation of dust grains and the thermal ionization of metals. The degree of magnetic decoupling is strongly influenced by the ionization rates, the grain parameters, and the abundance of metals (Ruzmaikina, 1985; Dudorov, 1986).

The results of the calculations show that the magnetic field strength inside the Sun in the T Tau stage is $10^{2}$ times lower than that of a frozen-in field. For a short period, after the Sun has arrived at the zero-age main sequence, the magnetic field may be of the order of $10^{5}-10^{6} \mathrm{G}$ at the core boundary, and $10^{4}-10^{5} \mathrm{G}$ near the base of the convection zone. The exponent in the relation $B \sim n^{k}$ is $k=0.57$ for the core.

\section{The dynamo process}

The subsequent evolution of the fossil magnetic field depends on hydromagnetic or resistive instabilities, buoyancy, and ohmic and turbulent diffusion. Convection in new-born stars at the $\mathrm{T}$ Tauri stage has a dual character: Inside stars with masses $M \approx 0.5-1.2 M_{\odot}$ convection is either absent at the pre-main sequence stage, with the consequence that the strength of the fossil magnetic field is diminished only slightly by ohmic decay, or turbulent convection converts the large-scale magnetic field into a small-scale one. The buoyancy of the magnetic flux tubes may cause the activity of the pre-main sequence stars.

On the other hand turbulent convection with differential rotation drives $\alpha \omega$-dynamos, which begin to operate after sufficient turbulent dissipation of the fossil magnetic field has taken place. The differential rotation is induced by gravitational compression of the protosun. At a nonlinear stabilization level the poloidal $\left(B_{p}\right)$ and toroidal $\left(B_{\varphi}\right)$ components of the magnetic field are connected with the dynamo number, $N_{D}=\alpha(\mathrm{d} \omega / \mathrm{d} r) R^{4} / \nu_{t}^{2}$, and with the turbulent velocity $V_{t}$ by the following relations (Dudorov et al., 1989):

$$
B_{p} B_{\varphi}=\pi \omega H_{p} V_{t}, \quad B_{\varphi}=N_{D}^{1 / 2} B_{p}
$$

where $\omega$ is the angular velocity, $H_{p}$ the pressure scale height. We can estimate $V_{t}$, assuming that the protosun luminosity $L$ is entirely determined by convective energy transport, which gives $V_{t}=\left(L / M \cdot H_{p}\right)^{1 / 3}$, where $H_{p}$ is the pressure scale height. This leads to

$$
\begin{aligned}
& B_{p}=\left(8 \pi H_{p} \rho / 3\right)^{1 / 3}\left(H_{p} / R\right)^{1 / 2}(\omega / R)^{3 / 4} V_{t}^{3 / 4}, \\
& B_{\varphi}=\left(24 \pi H_{p} \rho\right)^{1 / 2}\left(R / H_{p}\right)^{1 / 2}\left(\omega^{3} / R\right)^{1 / 4} V_{t}^{1 / 4} .
\end{aligned}
$$

For the standard solar model with a density near the core of $\rho=10 \mathrm{~g} \mathrm{~cm}^{-3}, B_{p}=10^{4}-10^{5}$ $\mathrm{G}$, and $B_{\varphi}=10^{5}-10^{6} \mathrm{G}$. At the base of the convection zone $\rho=0.1 \mathrm{~g} \mathrm{~cm}^{-3}, B_{p}=$ $3 \times 10^{3}-3 \times 10^{4} \mathrm{G}$, and $B_{\varphi}=3 \times 10^{4}-3 \times 10^{5} \mathrm{G}$. Such a field evolves with time to a state with $B_{\varphi}=B_{p}$.

When the Sun arrives at the main sequence, the internal convection ceases. Two scenarios are possible. In the first, the shrinking convection zone rises to the solar surface on the hydrodynamic time scale. In this case the magnetic field is retained inside the Sun. In the 
second scenario the convection is attenuated slowly on the hydrostatic time scale. In this case the convection, which preserves its turbulent character, may weaken the magnetic field. The magnetic field will then be reduced to $B_{p}=10-100 \mathrm{G}$ and $B_{\varphi}=100-1000 \mathrm{G}$ for $\rho=10 \mathrm{~g} \mathrm{~cm}^{-3}$, and $B_{p}=1-10 \mathrm{G}$ and $B_{\varphi}=10-100 \mathrm{G}$ for $\rho=0.1 \mathrm{~g} \mathrm{~cm}^{-3}$. The fossil magnetic field influences neither the internal structure of the Sun nor e.g. the oblateness of the solar core or the solar neutrinos, but it may change the spectrum of acoustic oscillations either directly, or by reducing the gradient of the angular velocity.

\section{The differential rotation and magnetic field}

It is difficult to estimate the value of the angular velocity gradient in stars at the $\mathrm{T}$ Tau stage. Therefore we assume that the differential rotation inside the Sun is represented by the observations of Duvall, Harvey, and Pomerantz (1986). We study the generation of a toroidal magnetic field by the observed differential rotation taking into account magnetic buoyancy, making use of the induction equation (1), where now $\mathbf{V}=\boldsymbol{\Omega} \times \mathbf{r}$ is the linear rotational velocity. Instead of $V_{A D}$ we need to insert the buoyancy velocity $V_{p}$ (Parker, 1979). An estimate shows that a stationary toroidal magnetic field establishes itself very quickly, having

$$
B_{\varphi}=\left[G B_{r} 8 \pi P\left(H_{p} / u_{T}\right)\left(R^{\prime} / L^{\prime}\right)^{2}\right]^{1 / 3}
$$

where $G=r \mathrm{~d} \omega / \mathrm{d} r, u_{T}=10 \mathrm{~cm} \mathrm{~s}^{-1}$ (Parker, 1979), $H_{p}$ and $L^{\prime}$ are the pressure and temperature scale heights, and $R^{\prime}$ the tube radius. At the core boundary $B_{\varphi}=3 \times 10^{7} \mathrm{G}$ and $10^{6} \mathrm{G}$, respectively, for the two scenarios of convective behaviour. The poloidal fossil magnetic field leads to the substantially larger value of $B_{\varphi}=3 \times 10^{8} \mathrm{G}$. Such a field is compatible with the virial theorem. One may obtain a great reduction of $B_{\varphi}$ if one assumes that differential rotation exists during some period.

Hydromagnetic instabilities lead to an upper limit for the toroidal field. The characteristic time scale for magnetic smoothing of the differential rotation, the Alfvénic time scale, is much shorter than the evolutionary time scale, even for the initial magnetic field of the protosolar cloud, $B_{p}=4.5 \times 10^{-4} \mathrm{G}$. Thus a significant and stable jump of the angular velocity is possible for a vanishing poloidal magnetic field, for instance on the surface of a magnetically insulating solar core. Small irregularities of the angular momentum may be influenced by torsional hydromagnetic waves between the core and the envelope, where the magnetic field lines should be rigidly fixed. In this case the core and the envelope of the Sun may be azimuthally shifted relative to each other by substantial angles. The characteristic period of oscillation is again the Alfvénic time scale (see also Rosner and Weiss, 1985; Gough, 1986; Moss, 1987; Mestel and Weiss, 1987).

\section{References}

Dudorov, A.E. (1986) 'The fossil magnetic field', Astron. Circular 1446, 1-8.

Dudorov, A.E. and Sazonov, Yu.V. (1981) 'Hydrodynamical collapse of interstellar magnetic clouds', Nauchn. Inf. Astron. Council USSR AN 49, 114-136. 
Dudorov, A.E., Krivodubskij, V.N., Ruzmaikina, T.V., and Ruzmaikin, A.A. (1989) 'Largescale internal magnetic field of the Sun', Astron. Zh. 66, No.4.

Duvall, T.L., Harvey, J.W., and Pomerantz, M.A. (1986) 'Latitude and depth variation of solar rotation', Nature 321, 500-501.

Gough, D.O. (1986) 'What causes the solar cycle', Nature 319, 263-264.

Mestel, L. and Weiss, N.O, (1987) 'Magnetic fields and non-uniform rotation in stellar radiative zones', Mon. Not. Royal Astron. Soc. 226, 123-135.

Moss, D. (1987) 'Internal magnetic field of the Sun', Mon. Not. Royal Astron. Soc. 224, 1019-1031.

Parker, E.N. (1979) Cosmical magnetic fields, Clarendon Press, Oxford.

Rosner, R. and Weiss, N.O. (1985) 'Differential rotation and magnetic torques in the Sun', Nature 317, 790-792.

Ruzmaikina, T.V. (1981) 'Magnetic field and turbulence in solar nebula' Adv. Space Res. COSPAR 1, 49-54.

Ruzmaikina, T.V. (1985) 'Magnetic field in collapsing presolar clouds', Astron. Vestnik 19, 101-108. 